\title{
Rhinobothryum bovallii (ANDERSSON, 1916) (SERPENTES: COLUBRIDAE): NUEVAS LOCALIDADES PARA COLOMBIA, DESCRIPCIÓN HEMIPENEAL Y COMENTARIOS SOBRE SU HISTORIA NATURAL.
}

\section{Rhinobothryum bovallii (Andersson, 1916) (Serpentes: Colubridae): new locations for Colombia, hemipeneal description and comments on its natural history.}

Luis Enrique Vera-Pérez ${ }^{1}$ Jorge Alberto Zúñiga-Baos², Esteban Alzate-Basto ${ }^{3}$

1. Biólogo Universidad del Cauca. Investigador independiente, Carrera 8 \# 2A-12 Barrio Las 2uintas, La Plata (Huila). luchoveraperez@gmail.com

2. Biólogo Universidad del Cauca. Investigador independiente.jorzuba@gmail.com

3. Docente instructor Facultad de Ciencias y Biotecnología, Universidad CES.lalzateb@ces.edu.co

\section{Resumen}

Se presentan nuevas localidades de la serpiente falsa coral Rhinobothryum bovallii en Colombia, dentro de las cuales se destaca el primer registro para el departamento del Huila y su ocurrencia dentro del ecosistema de bosque seco tropical. Adicionalmente, se proporciona una descripción complementaria de su estructura hemipeneal y datos sobre aspectos biológicos y ecológicos.

Palabras claves: atropellamiento vehicular, bosque seco tropical, hemipenes, serpiente falsa coral.

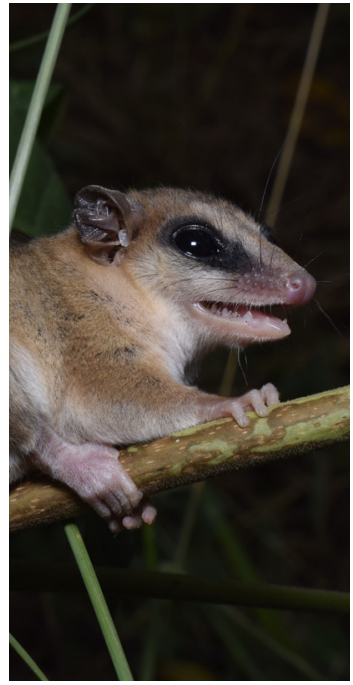

Historia del artículo

Fecha de recepción : $13 / 08 / 2019$

Fecha de aceptación: $28 / 10 / 2019$ 


\section{Abstract}

We present new locality records of the false coral snake Rhinobothryum bovallii in Colombia, highlighting the first record for the department of Huila, as well as its occurrence in the tropical dry forest ecosystem. Additionally, we provide a complementary description of its hemipenial structure and present data about biological and ecological aspects.

Key words: road kill, tropical dry forest, hemipenes, false coral snake.

\section{Introducción}

El género Rhinobothryum (Wagler, 1830), comprende las especies Rhinobothryum bovallii (Andersson, 1916) y Rhinobothryum lentiginosum (Scopoli, 1785), las cuales presentan una distribución disyuntiva que sugiere un proceso de especiación alopátrica (ver Rojas-Morales, 2012 para la discusión completa). Rhinobothryum bovallii se distribuye desde Centroamérica en Honduras, Nicaragua, Costa Rica y Panamá, hasta el norte de Suramérica en Colombia, Ecuador y Venezuela (Peters y Orejas-Miranda, 1970; Wallach et al., 2014; Uetz et al., 2019).

La distribución geográfica de $R$. bovallii fue actualizada recientemente por Martínez-Fonseca et al. (2019), quienes a través de diversas fuentes de información determinaron que en Colombia esta especie se encuentra en los departamentos de Antioquia, Boyacá, Caldas, Cauca, Cesar, Chocó, Córdoba, Magdalena, Santander,
Sucre y Valle del Cauca. Previamente, Rojas-Morales (2012) agrupó el conjunto de registros de $R$. bovallii dentro de la unidad biogeográfica del Chocó-Magdalena propuesta por Hernández-Camacho et al. (1992), ocupando ecosistemas de bosque lluvioso y posiblemente en bosques subandinos por debajo de los $1.000 \mathrm{msnm}$.

Esta serpiente es fácilmente distinguible de otras que poseen anillos de tres colores, por su coloración negra de escamas de la cabeza bordeadas de blanco o crema e hileras de escamas superiores quilladas posteriormente (Savage, 2002), que la hace inconfundible entre todas las especies de corales y falsas corales presentes en Colombia, excepto de $R$. lentiginosum, que se distingue por tener anillos negros considerablemente más largos y anillos rojos generalmente incompletos ventralmente (Martins y Oliveira, 1998). La morfología hemipeneal de la especie hasta hace poco era desconocida, sin embargo, MartínezFonseca et al. (2019) describieron los hemipenes parcialmente evertidos y no expandidos de un único ejemplar, concluyendo que en efecto son similares a los de R. lentiginosus. Esta conclusión estuvo basada en la descripción de Savage (2012), el cual a su vez utilizó la ilustración de un órgano retraído de Cope (1895), sin establecer diferencias entre los hemipenes de ambas especies.

\section{Materiales y métodos}

En el marco de las labores relacionadas con el manejo de fauna de los proyectos 
hidroeléctricos El Quimbo, Hidroituango y Porce III, sumado a registros ocasionales y resultados obtenidos por la Fundación Hidrobiológica George Dahl (FHGD)CORPOCESAR-PRODECO en los muestreos para la caracterización de fauna silvestre de la subcuenca del río Tucuy en el municipio de LaJagua de Ibirico (Cesar), se obtuvieron registros complementarios de la distribución de R. bovallii en Colombia. De estos registros, dos corresponden a individuos colectados que se encuentran depositados en la colección herpetológica del Museo de Historia Natural de la Universidad del Cauca (MHNUC).

Se examinó y caracterizó la estructura hemipeneal de los individuos colectados mediante sus órganos evertidos en el cuerpo y la extracción y eversión total con base en los métodos de preparación de Myers y Cadle (2003) y Zaher y Prudente (2003), expandiéndolo casi al máximo con la inyección de vaselina coloreada y luego teñido con rojo de alizarina para intensificar la visualización de las espinas calcáreas (Uzzell 1973). La morfología hemipeneal se describe de acuerdo a la terminología de Dowling y Savage (1960) y Zaher (1999).

\section{Resultados y discusión}

Se reporta por primera vez la presencia de $R$. bovallii para el departamento del Huila a través de un individuo capturado el 9 de abril de 2017 en el municipio de Gigante (Fig. 1A). Se obtuvo el segundo registro para la serranía del Perijá, en el municipio de La Jagua de Ibirico, departamento del

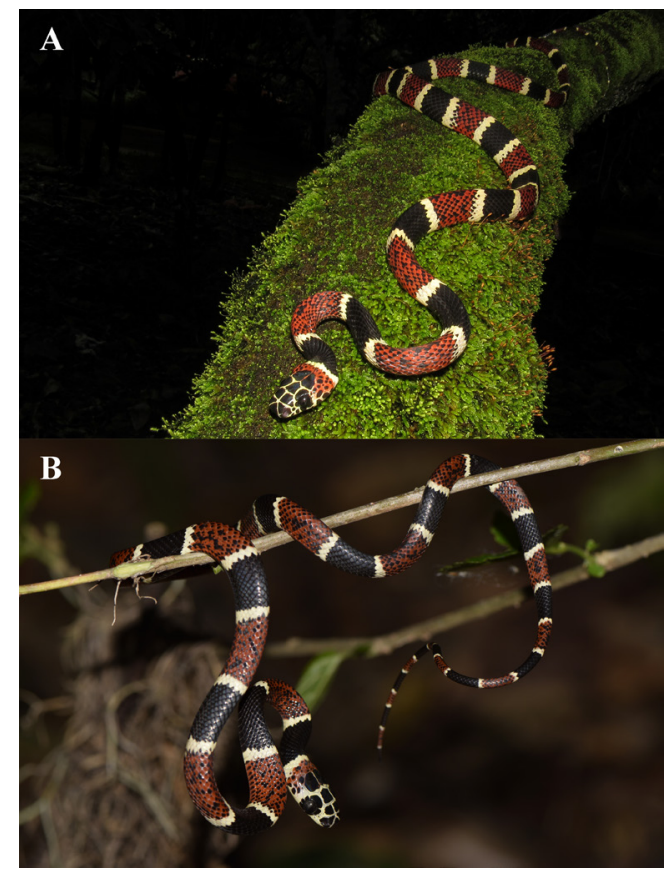

Figura 1. Rhinobothryum bovallii. Macho adulto del municipio de Gigante, Huila (A); juvenil del corregimiento La Victoria de San Isidro, municipio de La Jagua de Ibirico, Cesar (B). Fotografías de LEV-P.

Cesar (Fig. 1B). Además, se adicionan seis registros para el departamento de Antioquia, municipios de Toledo, Sabanalarga, Valdivia, Amalfi y Puerto Nare (Tabla 1).

Según estos registros junto con los presentados por Martínez-Fonseca et al. (2019), la distribución de $R$. bovallii en Colombia ya no es exclusiva para la provincia biogeográfica del Chocó-Magdalena (RojasMorales, 2012), sino que esta abarca también la norandina y el cinturón árido pericaribeño (ver Hernández-Camacho et al., 1992), siendo la localidad del departamento del 
Huila el registro más al sur en la cuenca del río Magdalena, mostrando una gran brecha entre este punto y los registros del Magdalena Medio (Fig. 2).

La comprobación de las coordenadas asignadas por Martínez-Fonseca et al.
(2019) revela una imprecisión para el punto localizado en el departamento del Cauca, el cual se ubica sobre la cuenca del río Cauca (parte alta) a una altitud aproximada de 1.700 msnm. Debido a la gran brecha de registros sobre esta cuenca hidrográfica, sumado a que todos los demás registros de esta especie

Tabla 1. Registros adicionales de Rhinobothryum bovallii en Colombia. Formato basado en Rojas-Morales (2012).

\begin{tabular}{|c|c|c|c|}
\hline Localidad & Georreferencia & Unidad biogeográfica & $\begin{array}{c}\text { Fuente de } \\
\text { datos }\end{array}$ \\
\hline $\begin{array}{l}\text { Departamento del Huila, } \\
\text { municipio de Gigante, vereda } \\
\text { La Honda }\end{array}$ & $\begin{array}{c}2^{\circ} 22 ' 10,6 ” \mathrm{~N} \\
75^{\circ} 34^{\prime} 18,2 ” \mathrm{~W} ; 725 \\
\text { msnm }\end{array}$ & $\begin{array}{l}\text { Provincia biogeográfica } \\
\text { norandina, distrito } \\
\text { Tolima }\end{array}$ & $\begin{array}{l}\text { MHNUC- } \\
\text { HE-Se-0693 } \\
\text { (Figura 1A) }\end{array}$ \\
\hline $\begin{array}{c}\text { Departamento del Cesar, } \\
\text { municipio de La Jagua de } \\
\text { Ibirico, corregimiento La } \\
\text { Victoria de San Isidro }\end{array}$ & $\begin{array}{c}9^{\circ} 35^{\prime} 42,8 ” \mathrm{~N} \\
73^{\circ} \mathrm{O} 9^{\prime} 31,9 " \mathrm{~W} ; 623 \\
\text { msnm }\end{array}$ & $\begin{array}{l}\text { Cinturón árido } \\
\text { pericaribeño, distrito } \\
\text { Ariguaní-Cesar }\end{array}$ & $\begin{array}{l}\text { obs. pers. } \\
\text { (Figura 1B) }\end{array}$ \\
\hline $\begin{array}{c}\text { Departamento de Antioquia, } \\
\text { municipio de Toledo, } \\
\text { corregimiento El Valle }\end{array}$ & $\begin{array}{c}7^{\mathrm{o}} \mathrm{O} 2{ }^{\prime} 28,4 " \mathrm{~N} \\
75^{\circ} 40^{\prime} 14,5^{\prime} \mathrm{W} ; 523 \\
\text { msnm }\end{array}$ & $\begin{array}{l}\text { Provincia biogeográfica } \\
\text { norandina, distrito } \\
\text { Cañón Cauca }\end{array}$ & $\begin{array}{l}\text { MHNUC- } \\
\text { HE-Se-O713 } \\
\text { (Figura 5) }\end{array}$ \\
\hline $\begin{array}{l}\text { Departamento de Antioquia, } \\
\text { municipio de Toledo, } \\
\text { corregimiento La Cascarela }\end{array}$ & $\begin{array}{c}7^{\circ} \mathrm{O} 5^{\prime} 09,3 " \mathrm{~N} \\
75^{\circ} 41^{\prime} 39,6 " \mathrm{~W} ; 381 \\
\text { msnm }\end{array}$ & $\begin{array}{l}\text { Provincia biogeográfica } \\
\text { norandina, distrito } \\
\text { Cañón Cauca }\end{array}$ & obs. pers. \\
\hline $\begin{array}{c}\text { Departamento de Antioquia, } \\
\text { municipio de Sabanalarga, } \\
\text { corregimiento Orobajo }\end{array}$ & $\begin{array}{c}7^{\circ} \mathrm{O} 1{ }^{\prime} 30, \mathrm{o}^{\prime} \mathrm{N} \\
75^{\circ} 47^{\prime} 35,5^{\prime} \mathrm{W} ; 394 \\
\text { msnm }\end{array}$ & $\begin{array}{l}\text { Provincia biogeográfica } \\
\text { norandina, distrito } \\
\text { Cañón Cauca }\end{array}$ & obs. pers. \\
\hline $\begin{array}{l}\text { Departamento de Antioquia, } \\
\text { municipio de Valdivia, vereda } \\
\text { La Habana }\end{array}$ & $\begin{array}{c}7^{\circ} 14^{\prime} 19,2 " \mathrm{~N}, \\
75^{\circ} 24^{\prime} 12,8^{\prime} \mathrm{W} ; 642 \\
\text { msnm aprox. }\end{array}$ & $\begin{array}{l}\text { Provincia biogeográfica } \\
\text { norandina, distrito } \\
\text { Bosques Subandinos } \\
\text { Cordillera Central }\end{array}$ & obs. pers. \\
\hline $\begin{array}{l}\text { Departamento de Antioquia, } \\
\text { municipio de Amalfi, vereda } \\
\text { María Teresa }\end{array}$ & $\begin{array}{l}6^{\mathrm{o}} 55^{\prime} 27,9 " \mathrm{~N} \\
75^{\circ} \mathrm{o} 8^{\prime} 33,1 " \mathrm{~W} ; 657 \\
\text { msnm aprox. }\end{array}$ & $\begin{array}{l}\text { Provincia biogeográfica } \\
\text { del Chocó-Magdalena, } \\
\text { distrito Nechí }\end{array}$ & $\begin{array}{c}\text { Suárez y } \\
\text { Alzate-Basto } \\
\quad(2014)\end{array}$ \\
\hline $\begin{array}{c}\text { Departamento de Antioquia, } \\
\text { municipio de Puerto Nare, } \\
\text { vereda Canteras }\end{array}$ & $\begin{array}{c}6^{\circ} 16^{\prime} 42,06 ” \mathrm{~N} \\
74^{\circ} 40^{\prime} 33,27^{\prime} \mathrm{W} ; 214 \\
\text { msnm aprox. }\end{array}$ & $\begin{array}{l}\text { Provincia biogeográfica } \\
\text { del Chocó-Magdalena, } \\
\text { distrito Nechí }\end{array}$ & obs. pers. \\
\hline
\end{tabular}


en Suramérica están por debajo de los 1.000 msnm (Rojas-Morales, 2012; Rojas-Runjaic e Infante-Rivero, 2018; Pazmiño-Otamendi, 2019), es probable que el espécimen AMNH R-107943 provenga de alguna localidad de la cuenca del Pacífico del departamento del Cauca, reduciendo la brecha de registros hacia el noroccidente del Ecuador (zonas tropical y subtropical occidental). Otra imprecisión menor se aprecia en la designación de las coordenadas del registro de ocurrencia IAvH:IAvH-R-3995 (BorjaAcosta, 2017), el cual proviene de "medio río Calima, 500 m” pero su asignación se ubica cerca al lago Calima a unos $1.500 \mathrm{msnm}$ aproximadamente.

El hemipene de $R$. bovallii es robusto, subcilíndrico y ligeramente cóncavo, unilobulado no capitado, con un surco espermático simple que se dirige por el centro del hemipene y termina en el final

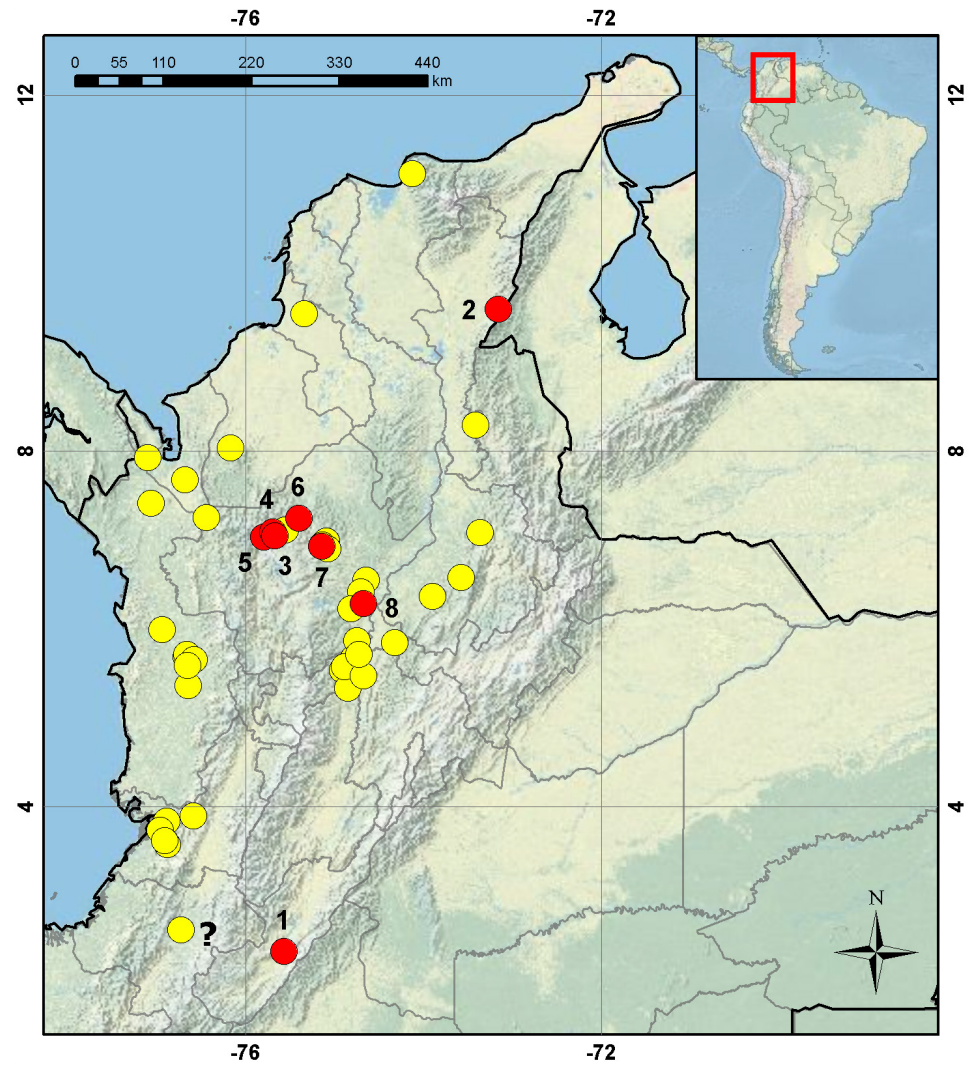

Figura 2. Distribución geográfica de Rhinobothryum bovallii en Colombia. Los puntos amarillos corresponden a las localidades reportadas por Martínez-Fonseca et al. (2019) y los puntos rojos a los nuevos registros referidos en la Tabla 1. El signo "?” en el departamento del Cauca, corresponde a una asignación dudosa. 
del capitulum (Fig. 3). La base del órgano es estriada y mayormente desnuda, con algunas espinas osificadas pequeñas; y posee un pequeño y profundo bolsillo acompañado por dos lóbulos a cada lado, uno de ellos muy prominente, plano y redondeado. El cuerpo está cubierto por espinas osificadas que son de mayor tamaño en los costados y la cara asulcada, y pequeñas a lo largo del surco espermático. El capitulum es tan largo como el cuerpo, redondeado y ornamentado con cálices que son espinulados en mayor proporción y papilados en la parte distal. Los cálices son más grandes y profundos en la región anterior de las caras laterales y asulcada.

De acuerdo a la descripción hemipeneal presentada aquí y en comparación con la información reportada por otros autores (Cope, 1895; Savage, 2002; MartínezFonseca et al., 2019), esta investigación coincide en que los órganos de las dos especies de Rhinobothryum son muy similares, específicamente en cuanto a su forma cilíndrica, sulco espermático simple, la presencia de espinas osificadas prominentes, lóbulo único y caliculado. Sin embargo, el órgano expandido y totalmente evertido de $R$. lentiginosum presentado por Montingelli et al. (2019: Fig. 3b), preparado con las mismas técnicas y de tamaño similar, permite una comparación más apropiada de la morfología hemipeneal de ambas especies. De acuerdo a esto, observamos que el órgano de $R$. bovallii en general es más robusto que el de su congénere, que el ápice del capitulum es completamente redondeado (vs levemente redondeado), y que la base del órgano de $R$. lentiginosum no posee un bolsillo profundo ni lóbulos prominentes. No obstante, estas diferencias que se proponen de manera preliminar deben ser evaluadas posteriormente mediante estudios que incluyan análisis de hemipenes de múltiples muestras.

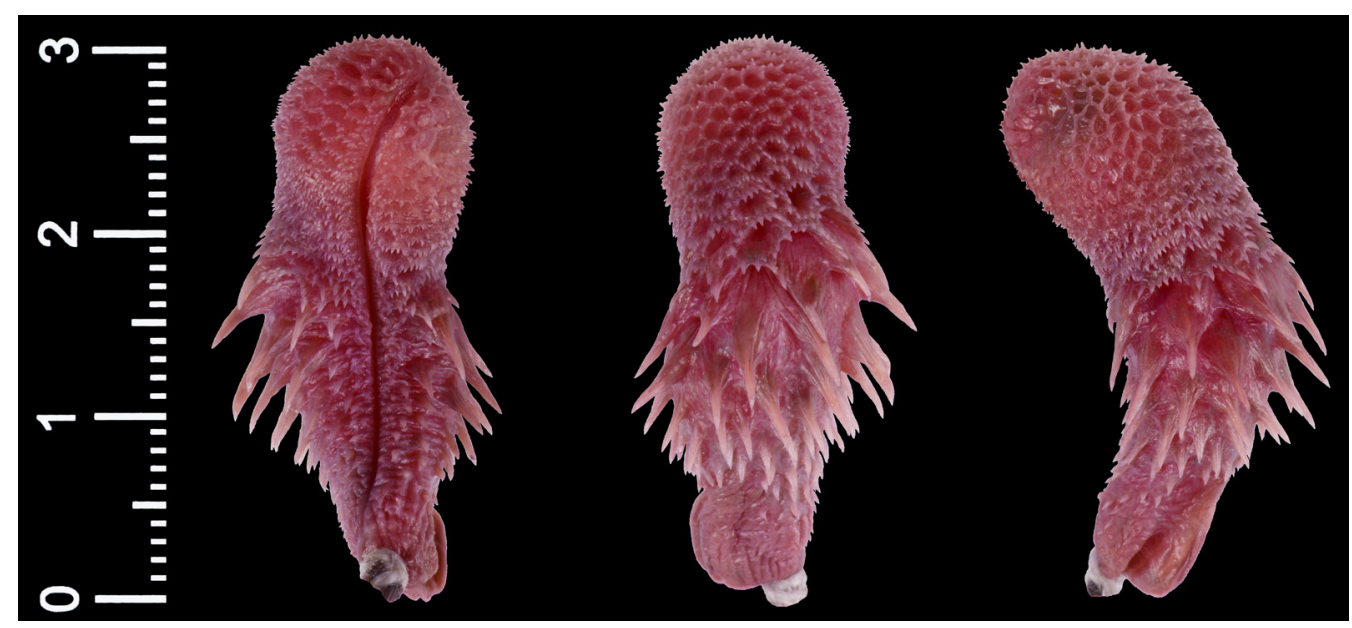

Figura 3. Hemipene izquierdo de Rhinobothryum bovallii (MHNUC-He-Se-o693) en vista sulcada (izquierda), asulcada (centro) y lateral (derecha). Escala en cm. Fotografías de LEV-P. 
Desde el registro en la localidad 1 , se consideró que la especie no solo ocupaba ecosistemas de bosque lluvioso y posiblemente bosques subandinos (RojasMorales, 2012), sino también el ecosistema de bosque seco tropical, pues a $350 \mathrm{~m}$ de este punto existía un polígono de más de 275 ha que fue transformado por el embalse El Quimbo. Lo mismo ocurría con las localidades 4 y 5 , situadas cerca a otras áreas de este ecosistema en Hidroituango. Finalmente, la presencia de esta especie en el bosque seco tropical se corroboró con el registro en la localidad 2 (Fig. 4), que corresponde a un polígono de 245,749 ha.

A pesar del gran avance en el conocimiento sobre aspectos biológicos y ecológicos de
R. bovallii (ver Pazmiño-Otamendi, 2019), coincidimos también con el criterio de otros autores con respecto a que es una especie raramente observada (Savage, 2002; Köhler, 2003; Rojas-Morales, 2012). Según Arredondo et al. (2017), esta baja tasa de encuentro puede estar relacionada con un esfuerzo de muestreo inadecuado, teniendo en cuenta que la especie es principalmente de actividad nocturna y de hábitos tanto terrestres como arbustivos que incluso alcanzan el dosel (Savage, 2002; PazmiñoOtamendi, 2019). De hecho, el individuo registrado en la localidad 2 (Tabla 1 ) fue observado mientras ascendía en un árbol de Ceiba sp. de gran porte, utilizando sus púas como soporte. Por otra parte, el individuo de la localidad 1 se encontró a 2,5 $\mathrm{m}$ de

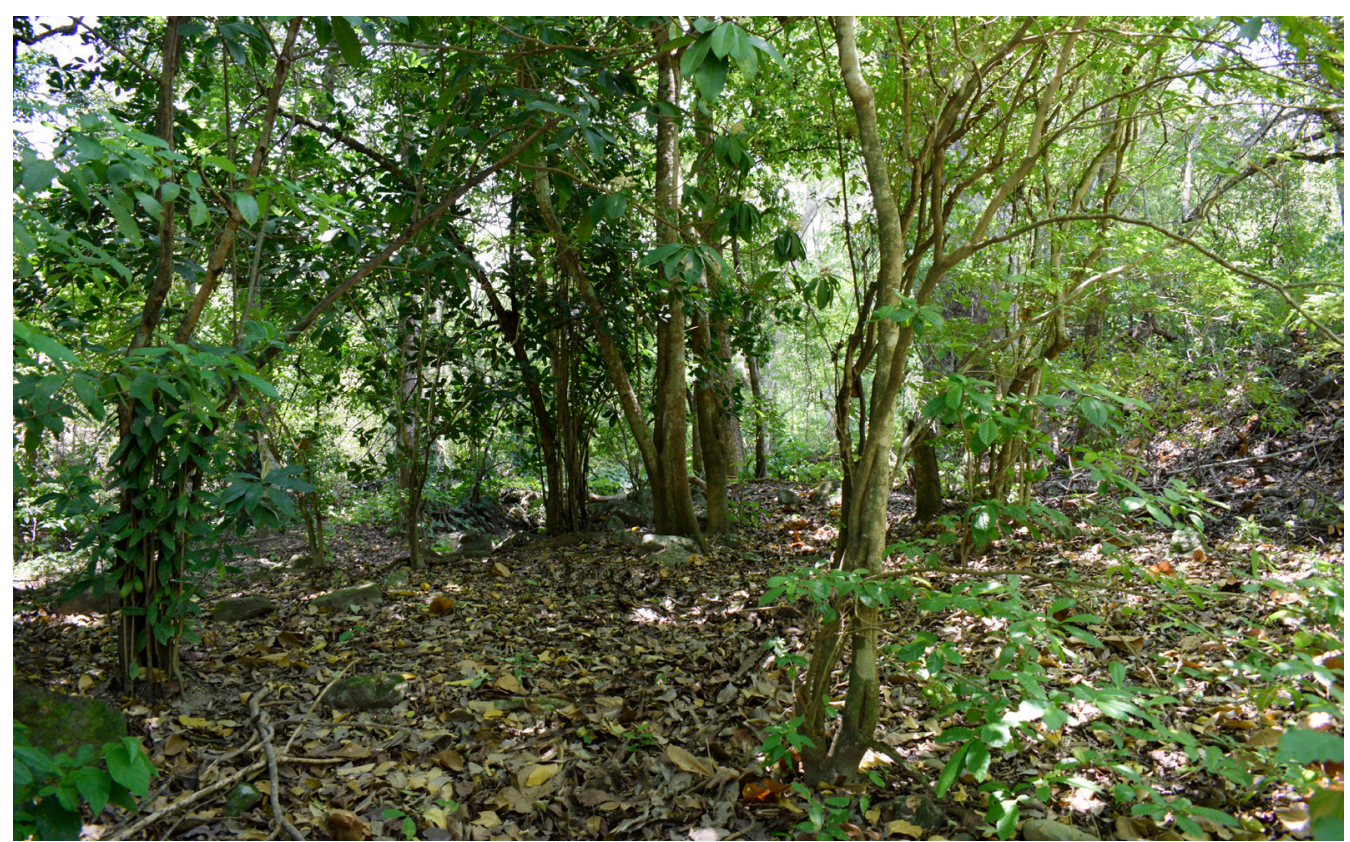

Figura 4. Bosque seco tropical en la vereda Zumbador Bajo, corregimiento La Victoria de San Isidro, municipio de La Jagua de Ibirico, Cesar. Fotografía de LEV-P. 
altura sobre un árbol de cacao (Theobroma cacao), en un cultivo pequeño y abandonado dentro del bosque de galería, donde existen árboles de gran talla como el caracolí (Anacardium excelsum).

La destrucción del hábitat representa una de las principales amenazas para la conservación de las serpientes en Colombia, y aunque pueden ser más tolerantes al disturbio que otros organismos, este proceso incrementa la tasa de encuentros con las comunidades humanas y, por ende, las matanzas directas, además de afectar en mayor proporción a organismos que pueden ser utilizados como presas (Lynch, 2012). Frente a esto, muchas serpientes se ven obligadas a incrementar su desplazamiento en búsqueda de mejores condiciones para la supervivencia, y en este proceso son víctimas del atropellamiento vehicular, una amenaza que produce una mortalidad calculada de 176,66o serpientes/año (Lynch, 2012). La especie $R$. bovallii se incluye dentro de las muchas que se ven afectadas por estas amenazas (Fig. 5), pues los registros de las localidades 3 y 6 corresponden a eventos de atropellamiento vehicular.

\section{Conclusiones}

El conocimiento de los aspectos biológicos y ecológicos de $R$. bovallii ha aumentado considerablemente en la presente década, especialmente en cuanto a lo relacionado con su distribución geográfica, sus relaciones filogenéticas y amenazas. Se espera que en

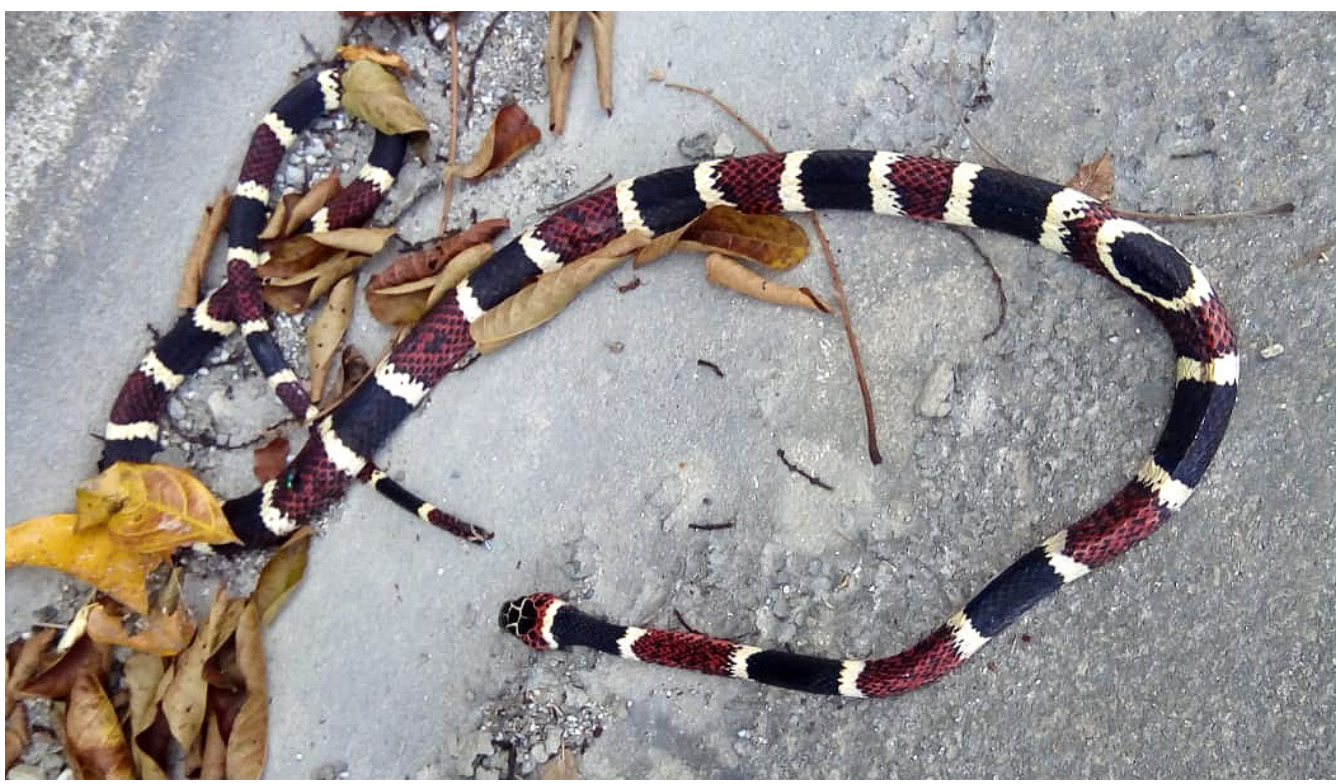

Figura 5. Individuo de Rhinobothryum bovallii (MHNUC-He-Se-Ooo713) atropellado entre el corregimiento El Valle y el municipio de Toledo, Antioquia. Foto de JAZ-B. 
un futuro próximo se registre esta especie en nuevas localidades Colombianas, especialmente en la región Caribe, la cuenca alta del río Magdalena, el chocó biogeográfico, y principalmente en los departamentos del Cauca y Nariño, dando continuidad a su distribución hacia el Ecuador.

\section{Agradecimientos}

LEV-P y JAZ-B agradecen a Santiago Ayerbe González y Raúl Rodríguez por el apoyo incondicional y la confianza brindada; y a la directora María Cristina Gallego Ropero, el profesor Jimmy Guerrero y el biólogo Andrés Felipe Liévano Bonilla por su amabilidad y diligencia en los procesos para el depósito de especímenes en el Museo de Historia Natural de la Universidad del Cauca. LEV-P agradece a Édgar Patiño y Martha Guerra por vincularlo a los muestreos en la FHGD.

\section{Bibliografía}

Arredondo, J. C., Cisneros-Heredia, D. F., Rivas, G., Sunyer, J. y Townsend, J. H. 2017. Rhinobothryum bovallii. The IUCN Red List of Threatened Species 2017: e.T177498A1489023. Fecha de acceso: 2019 ago. 13. Disponible en: http://dx.doi.org/10.2305/ IUCN.UK.2017-2.RLTS.T177498A1489023

Borja-Acosta, K. 2017. Rhinobothryum bovallii (Andersson, 1916). Colección de Reptiles del Instituto Alexander von Humboldt. Versión 23.o. Instituto de Investigación de Recursos Biológicos Alexander von Humboldt. Fecha de acceso: 2019 ago. 13. Disponible en: https://www.gbif.org/occurrence/1801434523

Cope, E. D. 1895. The classification of the Ophidia. Transactions of the American Philosophical Society, 18(2): 186-219. https:// archive.org/details/jstor-1005387

Dowling, H. G. y Savage, J. M. 1960. A guide to the snake hemipenis: A survey of basic structure and systematic characteristics. Zoologica, 45: 17-28. 
Hernández-Camacho, J., Hurtado-Guerra, A., Ortiz-Quijano, R. y Walschburger, T. 1992. Unidades biogeográficas de Colombia. Pp. 151-151. En: Halffter, G. (Ed.) La Diversidad Biológica de Iberoamérica. Acta Zoológica Mexicana, Volumen especial (n.s.), Xalapa,Veracruz, México. 390 pp.

Köhler, G. 2003. The reptiles of Central America. Herpeton Verlag, Offenbach, Alemania. 367 pp.

Lynch, J. D. 2012. El contexto de las serpientes de Colombia con un análisis de las amenazas contra su conservación. Revista de Academia Colombiana de Ciencias Exactas, Físicas y Naturales, 36(140): 435-449. http://www.scielo.org. co/pdf/racefn/v36n140/v36n140ao9.pdf

Martínez-Fonseca, J. G., Loza, J., Fernández, M., SalazarSaavedra, M. y Sunyer, J. 2019. First country record of Rhinobothryum bovallii (Anderson, 1916) (Squemata, Colubridae) from Nicaragua. Check List, 15(4): 555-563. https://checklist. pensoft.net/article/33987/

Martins, M. y Oliveira, M. E. 1998. Natural history of snakes in forests of the Manaus region, Central Amazonia, Brazil. Herpetological Natural History, 6(2): 78-150. http://eco.ib.usp. br/labvert/Martins\&Oliveira-HNH-1999.pdf

Montingelli, G. G., Grazziotin, F. G., Battilana,J., Murphy, R. W., Zhang, Y-P. y Zaher, H. 2019. Higher-level phylogenetic affinities of the Neotropical genus Mastigodryas Amaral, 1934 (Serpentes: Colubridae), species-group definition and description of a new genus for Mastigodryas bifossatus. Journal of Zoological Systematics and Evolutionary Research, 57(2): 205-239.

Myers, C. W. y Cadle, J. E. 2003. On the snake hemipenis, with notes on Psomophis and techniques of eversion: a response to Dowling. Herpetological Review, 34(4): 295-302. https://www. researchgate.net/publication/281621011_On_the_Snake_ Hemipenis_with_Notes_on_Psomophis_and_Techniques_ of_Eversion_A_Response_to_Dowling 
Pazmiño-Otamendi, G. 2019. Rhinobothryum bovallii. Reptiles del Ecuador. Version 2019.o. Museo de Zoología, Pontificia Universidad Católica del Ecuador. Fecha de acceso: 2019 ago. 13. Disponible en: https://bioweb.bio/faunaweb/reptiliaweb/ FichaEspecie/Rhinobothryum\%2obovallii

Peters, J. A. y Orejas-Miranda, B. 1970. Catalogue of the Neotropical Squamata: Part I. Snakes. United States National Museum Bulletin, 297: 1-347. https://www.biodiversitylibrary. org/page/7868973\#page/5/mode/1up

Rojas-Morales,J.A. 2012. On the geographic distribution of the false coral snake, Rhinobothryum bovallii (Serpentes: Dipsadidae), in Colombia - a biogeographical perspective. Salamandra, 48(4): 243-248. http://www.salamandra-journal.com/index.php/home/ contents/2012-vol-48/313-rojas-morales-j-a/file

Rojas-Runjaic, F. J. M. e Infante-Rivero, E. E. 2018. Redescubrimiento de las serpientes Rhinobothryum bovallii (Andersson, 1916) y Plesiodipsas perijanensis (Alemán, 1953) en Venezuela. Memoria de la Fundación La Salle de Ciencias Naturales, 76(184): 83-92. https://www.researchgate.net/ publication/328614468_Redescubrimiento_de_las_serpientes_ Rhinobothryum_bovallii_Andersson_1916_y_Plesiodipsas_ perijanensis_Aleman_1953_en_Venezuela

Savage, J. M. 2002. The amphibians and reptiles of Costa Rica. A herpetofauna between two continents, between two seas. University of Chicago Press, Chicago. 934 pp.

Uetz, P., Freed, P. y J. Hošek. 2019. The Reptile Database. Fecha de acceso: 2019 oct. 24. Disponible en: http://www. reptile-database.org

Uzzell, T. M. 1973. A revision of lizards of the genus Prionodactylus, with a new genus for $P$. lecostictus and notes on the genus Euspondylus (Sauria: Teiidae). Postilla, 159: 1-67. https://doi. org/10.5962/bhl.part.11535 
Wallach, V., Williams, K. L. y Boundy, J. 2014. Snakes of the World: A Catalogue of Living and Extinct Species. Taylor and Francis Group, CRC Press., Boca Raton, Florida. 1237 pp.

Zaher H. 1999. Hemipenial morphology of the South American Xenodontine snakes, with a proposal for a monophyletic Xenodontinae and a reappraisal of colubroid hemipenes. Bulletin of the American Museum of Natural History, 240: 1-168. http://digitallibrary.amnh.org/handle/2246/1646

Zaher, H. y Prudente, A. L. C. 2003. Hemipenes of Siphlophis (Serpentes, Xenodontinae) and Techniques of Hemipenial Preparation in Snakes: A Response to Dowling. Herpetological Review, 34(4): 302-307. https://www.researchgate.net/ publication/292954574_Hemipenes_of_Siphlophis_Serpentes Xenodontinae_and_Techniques_of_Hemipenial_Preparation_ in_Snakes_A_Response_to_Dowling 